
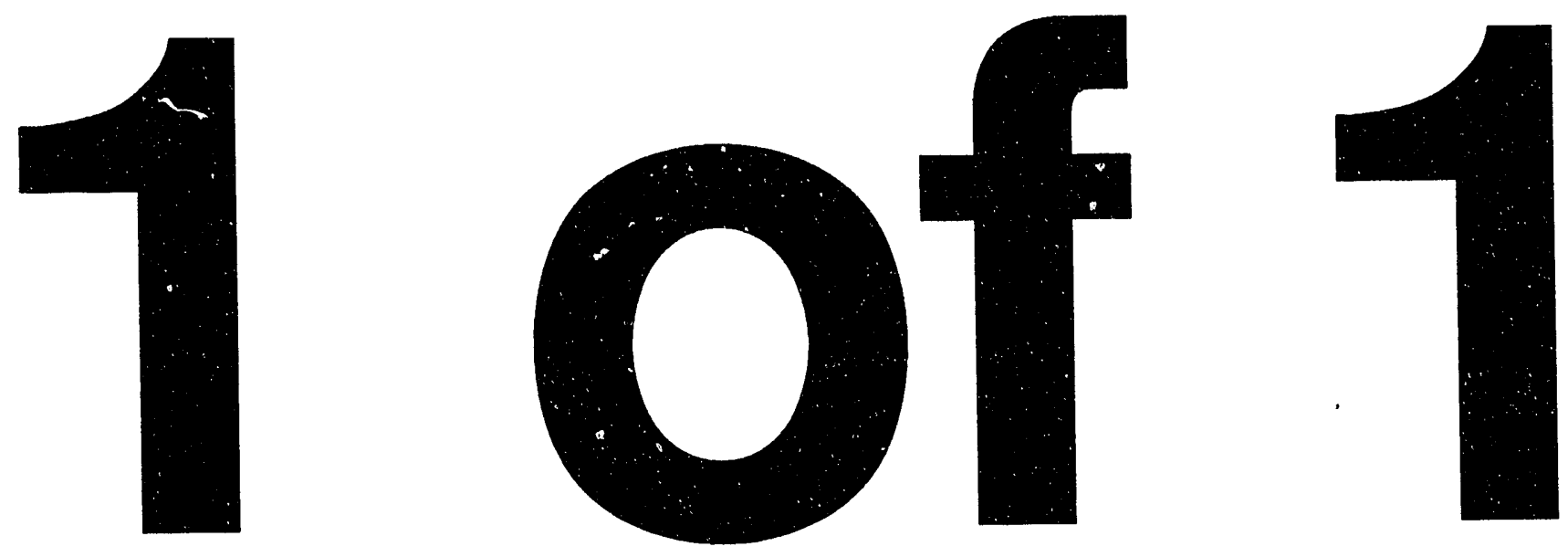
UC-420,427

BY

G.W. HAMMETT, M.A. BEER, W. DORLAND, ET AL.

JUNE, 1993

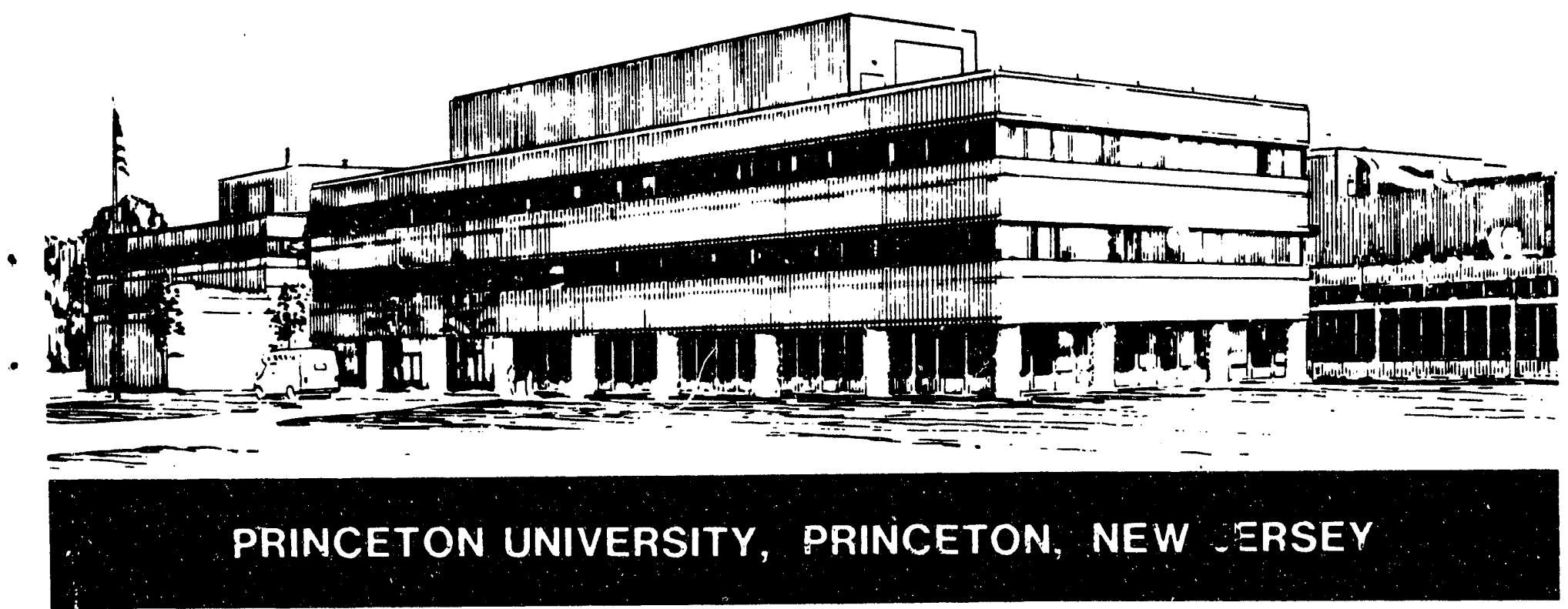

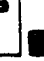




\section{NOTICE}

This report was prepared as an account of work sponsored by an agency of the United States Government. Neither the United States Government nor any agency thereof, nor any of their employees, makes any warranty, express or implied, or assumes any legal liability or responsibility for the accuracy, completeness, or usefulness of any information, apparatus, product, or process disclosed, or represents that its use would not infringe privately owned rights. Reference herein to any specific commercial produce, process, or service by trade name, trademark, manufacturer, or otherwise, does not necessarily constitute or imply its endorsement, recommendation, or favoring by the United States Government or any agency thereof. The views and opinions of authors expressed herein do not necessarily state or reflect those of the United States Government or any agency thereof.

\section{NOTICE}

This report has been reproduced from the best available copy.

Available in paper copy and microfiche.

Number of pages in this report: 15

DOE and DOE contractors can obtain copies of this report from:

Office of Scientific and Technical Information

P.O. Box 62

Oak Ridge, TN 37831;

(615) $576-8401$.

This report is publicly available from the:

National Technical Information Service

Department of Commerce

5285 Port Royal Road

Springfield, Virginia 22161

(703) $487-4650$ 


\title{
Developments in the Gyrofluid Approach to Tokamak Turbulence Simulations
}

\author{
G W Hammett, M A Beer, W Dorland, S C Cowley† \\ and S A Smith \\ Princeton University Plasma Physics Laboratory, \\ P.O. Box 451, Princeton, NJ 08543 USA \\ † Dept. of Physics, University of California, Los Angeles, CA 90024 USA
}

\begin{abstract}
A status report is given on recent developments in the gyrofluid approach to simulating tokamak turbulence. "Gyrofluid" (or "gyro-Landau fluid") equations attempt to extend the range of validity of fluid equations to a more collisionless regime typical of tokamaks, by developing fluid models of important kinetic effects such as Landaudamping and gyro-orbit averaging. The fluid moments approach should converge if enough moments are kept, though this may require a large number of moments for some processes. Toroidal gyrofluid equations have been extended from 4 to 6 moments, and to include the $\mu \nabla B$ magnetic mirroring force. An efficient field-line coordinate system for toroidal turbulence simulations (useful for both particle and fluid simulations) is presented. Nonlinear 3-D simulations of toroidal ITG-driven turbulence indicate that turbulence-generated sheared flows play an important role in the development and saturation of the turbulence. There is a strong enhancement of the flows when the electrons are assumed adiabatic on each flux surface, which is partially offset by toroidal drift effects which reduce the flows.
\end{abstract}

\section{Introduction}

Fluid equations have long been used to gain insight into plasma instabilities and turbulence. Our aim is to build on this by developing fluid models of important kinetic effects which are thought necessary for more realistic simulations of plasma turbulence in tokamaks. The "gyrofluid" equations provide the dynamics of a few moments (typically 4-6 moments, for density, parallel flow, parallel and perpendicular pressure, parallel and perpendicular heat flux, etc.) of the gyrokinetic equation, expressing fundamental nonlinear conservation laws which the turbulence must satisfy. Closure approximations for 
the highest moments are made which provide improved fluid models of kinetic effects such as wave-particle resonances (Landau-damping and its inverse) [1, 2], gyro-radius orbit-averaging[3], and the dominant nonlinearities[3]. [Sometimes they are called the "gyro-Landau" fluid equations to emphasize the Landau-damping model as well as the gyro-radius effects.] These equations provide $n$-pole approximations to the plasma dispersion function $Z$, and usually provide a fairly good approximation to the linear eigenfrequencies and eigenmodes for both unstable and damped modes. A nonlinear saturated state is achieved when the $\vec{E} \times \vec{B}$ nonlinearity (including FLR corrections) couples these various modes together. Nevertheless, a finite set of fluid moment equations represents an approximation which certainly breaks down in some regimes, and so some care must be exercised. Detailed comparisons between nonlinear gyrokinetic and gyrofluid simulations need to be carried out. Interesting things can be learned from both areas of agreement or disagreement, since much of our existing understanding about plasma turbulence is based on analogies with neutral fluid turbulence and on fluid-like models of plasmas (such as Hasegawa-Mima and Terry-Horton drift-wave models, Kadomtsev trapped-particle models, MHD, etc.).

In Sec. 2 we discuss how the fluid moments should converge if enough moments are kept, and how this sheds light on some processes which may not be adequately represented by just a few moments. Sec. 3 summarizes the recent extension of the gyrofluid equations to 6 moments in toroidal geometry, including the magnetic mirroring force. Sec. 4 describes a field-line coordinate system proposed by Cowley et al [4] (which has some similarities to the ballooning transformation) as an efficient way to represent short-wavelength turbulence. Sec. 5 presents results from toroidal nonlinear calculations which indicate the dominant role that turbulence-generated sheared flows have on the dynamics and saturation of the turbulence. In particular, the (usually) proper form of the adiabatic electron response causes a large enhancement in the perpendicular flows. The talk on which this paper is based included a discussion of nonlinear $\vec{E} \times \vec{B}$ phasemixing (a little-studied but potentially important nonlinear process) as an example of our approach to fluid models of phase-mixing. The reader interested in that topic is referred to [3].]

\section{Resolution limits and higher order Landau-fluid equations}

In our previous work $[1,2]$ we presented 2,3 , and 4-moment variations of tluid equations with models for Landau damping, and mentioned that one could extend this to an arbitrary number of moment equations, providing more information about the distribution function $f(v)$ and providing a more accurate $n$-pole approximation (where $n$ is the number of moments kept) of the plasma dispersion function $Z$. We have recently completed a study of the convergence properties of such higher order fluid moment equations, and have tested them on the nonlinear plasma echo problem. Details of this calculation will appear in a future paper[5], but we summarize some of the results here for the insight they provide on the resolution limits of a truncated set of fluid moment equations.

The mathematics for higher moments becomes more tractable if one uses Hermite polynomials in velocity when taking moments of the Vlasov equation, rather than using 
the usual $\int d v v^{\ell} \int$ or $\int d v(v-u)^{\ell} f$ fluid moments. The $\ell$ th Hermite moment is defined as

$$
\alpha_{\ell}(x, t)=\int d v H_{\ell}\left(\frac{v}{\sqrt{2} v_{t}}\right) f(x, v, t),
$$

where $v_{t}$ is a constant thermal velocity $\left(m v_{t}^{2}=T_{0}\right)$ which sets the velocity scale, and $H_{\ell}$ is the fth Hermite polynomial,

$$
H_{\ell}(v)=(-1)^{\ell} e^{v^{2}} \frac{d^{\ell}}{d v^{\ell}} e^{-v^{2}} .
$$

The first $\ell$ Hermite moments and the first $\ell$ fluid moments contain exactly the same information, since the polynomials $1, v,(v-u), \ldots,(v-u)^{\ell}$ span the same space as $H_{0}(v), H_{1}(v), \ldots, H_{\ell}(v)$. The orthogonality properties of the Hermite polynomials then allow us to expand $f$ in a Hermite polynomial series:

$$
f(x, v, t)=\frac{1}{\sqrt{2 \pi} v_{t}} e^{-v^{2} / 2 v_{t}{ }^{2}} \sum_{\ell=0}^{\infty} \frac{\alpha_{\ell}(x, t)}{2^{\ell} \ell !} H_{\ell}\left(\frac{v}{\sqrt{2} v_{t}}\right) .
$$

This provides a direct link between the "fluid" approach of evolving $n$ fluid moment equations, and the "kinetic" approach of solving the Vlasov equation with $n$ Hermite polynomials. The usage of Hermite polynomials for solving the Vlasov equation was introduced independently by Armstrong et al [6], Grant and Feix[7], and by Sadowski[8]. This approach was used to study a number of early plasma problems (linear and nonlinear Landau damping, two-stream instabilities, plasma echoes, etc.). The Hermite polynomials provide a complete basis set, and so should converge if enough moments are kept. As pointed out on p.67 of the review article by Armstrong[6], the nature of the resolution limits of a truncated Hermite expansion can be illustrated by just considering the free streaming equation

$$
\frac{\partial f}{\partial t}+v \frac{\partial f}{\partial x}=0
$$

We will compare Hermite-based solutions of this with the exact solution, which is just

$$
f=f_{M}(v) e^{i k(x-v t)}
$$

for the initial condition $f=f_{M}(v) e^{i k x}$, where $f_{M}$ is a Maxwellian. Even though $f$ is initially a smooth function of velocity, it eventually becomes a very oscillatory function of $v$ for large enough $t$ (this is responsible for the phase-mixing decay of the density $\left.n=\int d v f\right)$, and therefore requires a large number of Hermite polynomials to accurately represent it.

Operating on Eq. (4) with $\int d v H_{\ell}$, and using the recursion relation $H_{\ell+1}(x)=$ $2 x H_{\ell}(x)-2 \ell H_{\ell-1}(x)$, yields

$$
\frac{\partial \alpha_{\ell}}{\partial t}+\sqrt{2} v_{t}\left(\ell \frac{\partial \alpha_{\ell-1}}{\partial x}+\frac{1}{2} \frac{\partial \alpha_{\ell+1}}{\partial x}\right)=0 .
$$

Like the usual fluid moments equations, this is an infinite set of coupled equations, each expressing a conservation law, but which requires some closure approximation in 
practice, and one must consider resulting truncation errors. Taking a time derivative of Eq. (6), Fourier transforming in $x$, and normalizing time to $1 /\left(k_{x} v_{t} \sqrt{2}\right)$, leads to

$$
\frac{\partial^{2} \alpha_{\ell}}{\partial t^{2}}+\ell(\ell-1) \alpha_{\ell-2}+\left(\ell+\frac{1}{2}\right) \alpha_{\ell}+\frac{1}{4} \alpha_{\ell+2}=0
$$

Scaling the coefficients by $\alpha_{2 \ell}(t)=(-1)^{\ell_{2} \ell}(2 \ell-1) ! ! x_{\ell}(t)$ (note the factor of 2 difference in the $\alpha$ and $x$ indices), it is possible to rewrite this in the form

$$
M_{\ell} \frac{d^{2} x_{\ell}}{d t^{2}}=k_{\ell+1}\left(x_{\ell+1}-x_{\ell}\right)-k_{\ell}\left(x_{\ell}-x_{\ell-1}\right) .
$$

I.e., this provides a precise mechanical analog to Eqs.(6) in terms of a infinite set of masses $M_{\ell}$ at positions $x_{\ell}$ (for $\ell=0,1,2, \ldots$ ) each coupled to its nearest neighbors $M_{\ell-1}$ and $M_{\ell+1}$ by springs with spring constant $k_{\ell}$ and $k_{\ell+1}$. (A solution for the coefficients is $k_{\ell}=\ell M_{\ell}$ and $M_{\ell}=(2 \ell) ! /\left(4^{\ell}(\ell !)^{2}\right) \sim 1 / \sqrt{\pi \ell}$.) An initial density perturbation, i.e., an initial perturbation in the lowest moment $\alpha_{0}$, can be thought of as a perturbation in the position $x_{0}$ of the first mass in the mechanical system. This then produces a wave in the coupled springs which propagates to high $\ell$. One can take Hermite moments of the exact solution $f=f_{M}(v) \exp (i k(x-v t))$ and show that [6]

$$
\alpha_{\ell}(t) \propto t^{\ell} e^{-\left(k v_{t} t\right)^{2} / 2}
$$

The peak of the wave thus reaches the $\ell^{\prime}$ th moment (i.e., the $\ell / 2$ mass) at $t=\sqrt{\ell} /\left(k v_{t}\right)$. After that time, most of the energy in the wave will have propagated to higher $\ell$ 's. Truncating the Hermite expansion by setting $\alpha_{L}=0$ corresponds to replacing the $L / 2$ mass with a fixed wall (i.e., setting $x_{L / 2}=0$ ) which reflects the energy back to lower springs (i.e., to lower moments). There is no damping mechanism in this mechanical system, so the wave energy is trapped and bounces around between $\ell=0$ and $\ell=$ $L / 2-1$. The density $\left(\alpha_{0}\right)$ will appear to phase-mix for short times as the wave energy propagates to higher $\ell$ 's, but the system has no dissipation in it, and it will periodically reconstruct the initial perturbation (see Fig. 1 in Ref.[2] for examples of this). To rectify this problem, the early computational studies[6] would sometimes include a weak amount of velocity-space diffusion (which might arise either from particle collisions or from wave-induced quasilinear diffusion) in the Vlasov equation. For example, using the Lenard-Bernstein model collision operator[9], $C(f)=\partial / \partial v\left(\nu v f+\nu v_{t}^{2} \partial f / \partial v\right)$, adds the term $-\nu \ell \alpha_{\ell}$ to the right-hand-side of Eq. (6), thus providing larger damping at higher moments. For low collisionality problems, this requires a large number of Hermite moments (and the stiffness of the equations increases as more moments are kept). The early computational studies[6] frequently kept ten's or hundred's of Hermite moments.

In the framework of the above mechanical analogy, our fluid model of Landau damping $[1,2]$ can be interpreted as providing damping for the highest- $\ell$ spring in the system to reduce artificial reflection of the wave back to the lower springs. I.e., our closure approximation for the highest moment $\alpha_{L}$ introduces damping into the equation for $\partial \alpha_{L-1} / \partial t$ which models the rate at which energy is flowing from the resolved lower moments $\ell<L$ to unresolved higher moments $\ell \geq L$, where it is presumed to be eventually damped at high enough $\ell$ by collisions. This simplifies the problem and allows the use of fewer moments than would be required if one had to resolve all the 
way up to the collisional scale. While this assumption works in many cases, there are certainly some cases where it breaks down, where some additional effect causes the information to be reflected back down to low $\ell$ 's before the information had propagated to high enough $\ell$ 's to be damped. One example of this is the nonlinear plasma echo.

In the classic plasma echo problem $[10,11]$, one considers the response of the plasma to an electric field of the form $E=E_{1} \cos \left(k_{1} x\right) \delta(t)+E_{2} \cos \left(k_{2} x\right) \delta\left(t-t_{2}\right)$. Linearly these two electric pulses produce density perturbations at wavelengths $k_{1}$ and $k_{2}$ which then decay in time due to phase-mixing. The nonlinear interaction between the $E_{2}$ pulse and the $f_{1} \propto E_{1} v f_{M}(v) \cos \left(k_{1}(x-v t)\right)$ produces a perturbation in $f$ at the wavenumber $k_{2}-k_{1}$ which "un-phase-mixes" in time producing a maximum density perturbation (an "echo") around the time $t_{e c h o}=t_{2} k_{2} /\left(k_{2}-k_{1}\right)$. Numerical solutions of Landau-fluid equations are easily able to reproduce the phase-mixing decay of the linear perturbations at $k_{1}$ and $k_{2}$ even with just a few (2-4) moments (as they were designed to do), however we have found that one must keep of order $\left(k_{1} v_{t} t_{2}\right)^{2}$ moments in order to reproduce the echo (details of this calculation will be reported later[5]). This is consistent with the number of Hermite moments needed to represent the highly oscillatory $f_{1}$ at the time $t_{2}$ of the second pulse.

Ion Compton scattering (ICS) is another process which may, in some cases, require more moments. Mattor[12] has carried out a weak-turbulence analysis of a simplified version of gyro-Landau fluid equations (with 3 moments). The range of validity of weak-turbulence theory for ITG modes is fairly narrow [13], but it does provide a useful analytic test of nonlinear effects. The 3-moment fluid approach successfully[12, 14] reproduces ion Compton scattering for drift-waves with $\omega \gg k_{\|} v_{t}$. However, for ITG modes very near marginal stability, with low frequencies $\omega \ll k_{\|} v_{t}$, Mattor found that the ICS rate predicted by the fluid approach is too small by a factor of $\left(\omega / k_{\|} v_{t}\right)^{2}$. Thus one needs to be cautious with Landau-fluid equations in cases where significant ICS might be expected. Based on the above discussion about resolution limits of Hermite polynomial representations, one might conjecture that in order to properly reproduce ICS one needs to follow the evolution of $f_{1} \propto \exp \left(i k_{\|}\left(z-v_{\|} t\right)\right)$ for a time $t \sim 1 / \omega$, so that the number of moments needed might scale as $L \sim\left(k_{\|} v_{t} t\right)^{2} \approx\left(k_{\|} v_{t} / \omega\right)^{2}$. This is a large number in the particular regime of near-marginal stability for low-frequency ITG modes investigated by Mattor. Further from marginal stability, one frequently finds that the fastest growing ITG modes (which may or may not dominate the nonlinear spectrum) typically have $\omega \sim k_{\|} v_{t}$, so that perhaps a few moments will be sufficient (as suggested by the successful drift wave results where $k_{\|} v_{t} / \omega$ was small). These are conjectures, however, and further study of these issues should be carried out.

\section{Toroidal Gyrofluid Equations}

The toroidal gyrofluid equations are derived by taking velocity moments $\int d^{3} v v_{\|}^{n} v_{\perp}^{2 m}$ of the nonlinear electrostatic gyrokinetic equation in toroidal geometry: $[15,16,17]$

$$
\begin{aligned}
& \frac{\partial}{\partial t} F B+\nabla \cdot {\left[F B\left(v_{\|} \hat{\mathbf{b}}+J_{0} \mathbf{v}_{E}+\mathbf{v}_{d}\right)\right] } \\
&+\frac{\partial}{\partial v_{\|}}\left[F B\left(-\frac{e}{m} \hat{\mathbf{b}} \cdot \nabla J_{0} \Phi-\mu \hat{\mathbf{b}} \cdot \nabla B+v_{\|}(\hat{\mathbf{b}} \cdot \nabla \hat{\mathbf{b}}) \cdot J_{0} \mathbf{v}_{E}\right)\right]=C(F) .
\end{aligned}
$$


The toroidal effects are contained in $\mathbf{v}_{d}$ (the curvature and $\nabla B$ drifts), a modification to the parallel acceleration (the $\hat{\mathbf{b}} \cdot \nabla \hat{\mathbf{b}}$ term), the mirroring force $\mu \hat{\mathbf{b}} \cdot \nabla B$, and the non-zero divergence of $\mathbf{v}_{E}=c \vec{E} \times \vec{B} / B^{2}$ in toroidal geometry. The highest moments introduced are approximated in terms of lower moments to accurately model the linear kinetic response. In the parallel terms, this is accomplished by adding damping proportional to $\left|k_{\|}\right|,[1]$ while in the toroidal terms the damping is proportional to $|\omega, d| \cdot[18]$ For the $\mu \hat{\mathbf{b}} \cdot \nabla B$ terms, the higher moments are approximated as Maxwellian perturbations, without dissipation. These terms incorporate trapped particle effects, reproducing the C'GL pressure balance equation. The collision operator is modeled by a number, momentum, and energy conserving Krook operator, ignoring FLR corrections which give rise to classical transport, but roughly capturing neoclassical effects from ion-ion collisions $\left(\nu_{i i}\right)$. Taking moments of the $J_{0} \Phi$ terms introduces FLR approximations (not shown here) as discussed in [3]. We summarize these equations here; a complete derivation will be presented in a future paper.

$$
\begin{aligned}
\frac{d n}{d t}+ & B \hat{\mathbf{b}} \cdot \nabla \frac{u_{\|}}{B}-i \omega_{*} \Phi+i \omega_{d}\left(\frac{p_{\|}+p_{\perp}}{2}+\Phi\right)=0 \\
\frac{d u_{\|}}{d t}+ & B \hat{\mathbf{b}} \cdot \nabla \frac{p_{\|}}{B}+\hat{\mathbf{b}} \cdot \nabla \Phi+\frac{p_{\perp}}{B} \hat{\mathbf{b}} \cdot \nabla B+i \omega_{d}\left(\frac{q_{\|}+q_{\perp}}{2}+2 u_{\|}\right)=0 \\
\frac{d p_{\|}}{d t}+ & B \hat{\mathbf{b}} \cdot \nabla\left(\frac{q_{\|}+3 u_{\|}}{B}\right)+2\left(\frac{q_{\perp}+u_{\|}}{B}\right) \hat{\mathbf{b}} \cdot \nabla B-i \omega_{*}(1+\eta) \Phi \\
& +i \omega_{d} \frac{1}{2}\left(7 p_{\|}+p_{\perp}-4 n+4 \Phi\right)+\left|\omega_{d}\right|\left(\nu_{1} T_{\|}+\nu_{2} T_{\perp}\right)=-\frac{2}{3} \nu_{i \mathfrak{i}}\left(p_{\|}-p_{\perp}\right) \\
\frac{d p_{\perp}}{d t} & +B^{2} \hat{\mathbf{b}} \cdot \nabla\left(\frac{q_{\perp}+u_{\|}}{B^{2}}\right)-i \omega_{*}(1+\eta) \Phi \\
& +i \omega_{d} \frac{1}{2}\left(5 p_{\perp}+p_{\|}-3 n+3 \Phi\right)+\left|\omega_{d}\right|\left(\nu_{3} T_{\|}+\nu_{4} T_{\perp}\right)=\frac{1}{3} \nu_{i i}\left(p_{\|}-p_{\perp}\right) \\
\frac{d q_{\|}}{d t} & +\left(3+\beta_{\|}\right) \hat{\mathbf{b}} \cdot \nabla T_{\|}+\sqrt{2} B D_{\|}\left|k_{\|}\right| \frac{q_{\|}}{B} \\
& +i \omega_{d} \frac{1}{2}\left(-3 q_{\|}-3 q_{\perp}+6 u_{\|}\right)+\left|\omega_{d}\right|\left(\nu_{5} u_{\|}+\nu_{6} q_{\|}+\nu_{7} q_{\perp}\right)=-\nu_{i i} q_{\|} \\
\frac{d q_{\perp}}{d t} & +B^{2} \hat{\mathbf{b}} \cdot \nabla \frac{T_{\perp}}{B^{2}}+\sqrt{2} B^{2} D_{\perp}\left|k_{\|}\right| \frac{q_{\perp}}{B^{2}}+\left(\frac{3 T_{\perp}-T_{\|}}{B}\right) \hat{\mathbf{b}} \cdot \nabla B \\
& +i \omega_{d} \frac{1}{2}\left(-q_{\|}-q_{\perp}+u_{\|}\right)+\left|\omega_{d}\right|\left(\nu_{8} u_{\|}+\nu_{9} q_{\|}+\nu_{10} q_{\perp}\right)=-\nu_{i i} q_{\perp} \\
\frac{d}{d t}= & \frac{\partial}{\partial t}+\mathbf{v}_{E} \cdot \nabla+\mathrm{FLR} \text { Corrections } \quad i \omega_{d}=-\frac{2 \rho_{i} v_{t i}}{R}\left[\frac{\cos \theta}{r} \frac{\partial}{\partial \theta}+\sin \theta \frac{\partial}{\partial r}\right]
\end{aligned}
$$

The parallel closure coefficients are $D_{\|}=2 \sqrt{\pi} /(3 \pi-8), \beta_{\|}=(32-9 \pi) /(3 \pi-8)$, $D_{\perp}=\sqrt{\pi} / 2$. The toroidal closure coefficients are of the form $\nu=\left(\nu_{r}, \nu_{i}\right)=\nu_{r}+$ $i \nu_{i}\left|\omega_{d}\right| / \omega_{d}$, where $\nu_{1}=(1.9,-1.3), \nu_{2}=(.43,1.2), \nu_{3}=(-.46,1.1), \nu_{4}=(-.10,-1.5)$, $\nu_{5}=(-8.1,12.6), \nu_{6}=(6.4,13.0), \nu_{7}=(7.6,6.2), \nu_{8}=(3.5,-7.0), \nu_{9}=(3.4,-4.9)$, $\nu_{10}=(8.9,-6.7)$. This large number of coefficients is due to the fact that the toroidal drifts introduce $v_{\|}^{4}, v_{\|}^{2} v_{\perp}^{2}, v_{\perp}^{4}, v_{\|}^{5}, v_{\|}^{3} v_{\perp}^{2}$, and $v_{\|} v_{\perp}^{4}$ moments. The gyrokinetic quasineutrality constraint is discussed in Sec. 5 . The gyrofluid model agrees well with the linear 


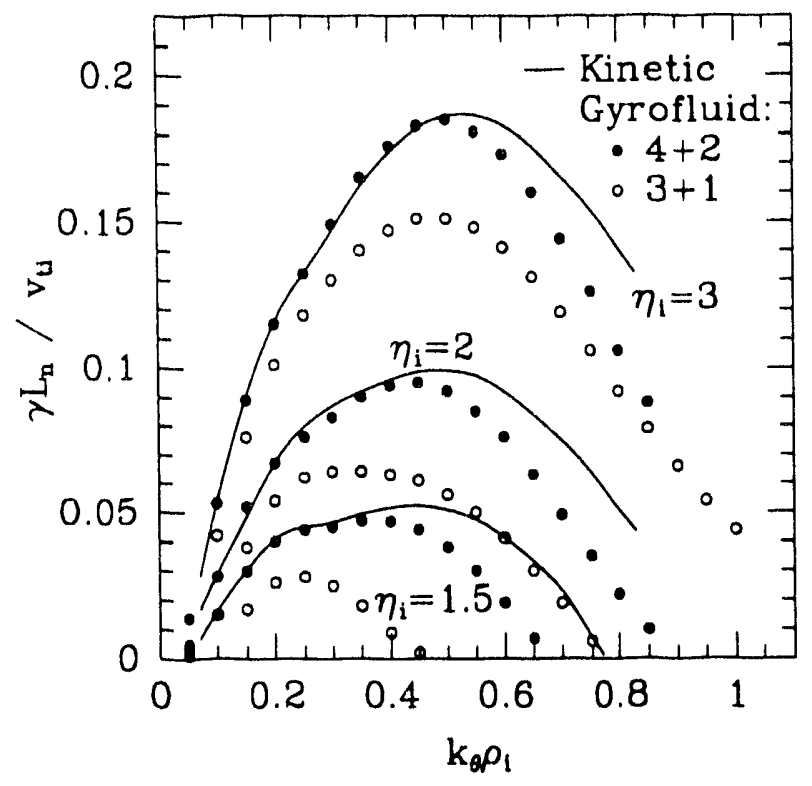

Figure 1. Comparison of linear growth rates with the kinetic ballooning results of Ref.[19] for the toroidal ITG mode for $q=2.4, \hat{s}=1, L_{n} / R=0.2$, $T_{i}=T_{e}$. The solid circles are with the new 6-moment equations, the open circles are with 4-moment equations and the closure in Ref.[18].

growth rates (and eigenmode structure) from fully kinetic integral calculations[19] of the toroidal ITG mode (assuming $\hat{\mathrm{b}} \cdot \nabla B=0$ ), as shown in Fig. 1.

\section{An efficient field-line coordinates representation}

Cowley et al [4] proposed the use of a field-line coordinate system as an efficient and natural geometry for tokamak turbulence simulations. This approach has advantages for both particle and fluid simulations. The basic idea is to take advantage of the short perpendicular correlation lengths of tokamak turbulence, while still allowing for long parallel correlation lengths and rapid parallel propagation, by simulating a thin flux tube which is extended along the field lines. The most efficient computation possible would use the smallest simulation volume necessary. I.e., the size of the simulation volume may only need to be a few decorrelation lengths in each direction, and it would seem unnecessary to simulate the whole tokamak to reproduce small-scale, locallydriven turbulence. [Ultimately, this hypothesis should be tested by varying the size of the simulation region to see if the character of the turbulence is indeed independent of the box size once the box is large enough. Also, the assumption of "locally-driven" turbulence is another important caveat which may need investigation. An analogy might be drawn with the ocean, where waves 20 miles apart are statistically uncorrelated (they have random phases), yet the average amplitude of the waves on the beach will be larger if there is a storm 20 miles out at sea. It is usually thought that magnetic shear, the radial variation of $\omega_{*}$, and other effects probably localize tokamak turbulence, but 
experiments and nonlinear theoretical investigations of this may be interesting.] The use of a field-line following coordinate system also allows the use a coarse grid in the parallel direction, providing further computational savings.

BES measurements in the TFTR experiment[20] indicate that the perpendicular correlation lengths are of order $2 \mathrm{~cm}$ (for a particular set of experimental conditions). Though this is much longer than the ion gyroradius $\rho_{i} \sim 0.15 \mathrm{~cm}$, it is still much smaller than the $80 \mathrm{~cm}$ plasma minor radius, so it would seem unnecessary to simulate the whole plasma to reproduce this short-scale phenomena. We have done simulations in a flux tube of dimensions $9 \mathrm{~cm}$ radial $\times 15 \mathrm{~cm}$ pol..ial $\times 2 \pi q R$ along the field line, i.e., the simulation box is $4-7$ times wider than the experimentally measured decorrelation length. Some initial nonlinear results are reported in the next section.

The essential features of the field-line approach recommended by Cowley et al [4] can be illustrated in a simple sheared slab geometry with the magnetic field $\vec{B}=$ $B_{0}(\hat{z}+s x \hat{y})$. Define the coordinate transformation

$$
x^{\prime}=x, \quad y^{\prime}=y-s x z, \quad z^{\prime}=z .
$$

Using the chain rule, derivatives are transformed by

$$
\frac{\partial}{\partial x}=\frac{\partial}{\partial x^{\prime}}-s z^{\prime} \frac{\partial}{\partial y^{\prime}}, \quad \frac{\partial}{\partial y}=\frac{\partial}{\partial y^{\prime}}, \quad \frac{\partial}{\partial z}=\frac{\partial}{\partial z^{\prime}}-s x^{\prime} \frac{\partial}{\partial y^{\prime}} .
$$

In the new primed coordinates, magnetic field lines are straight and are labeled by a particular value of $\left(x^{\prime}, y^{\prime}\right)$. Derivatives along the field line become simply $\hat{b} \cdot \nabla=(\hat{z}+$ $s x \hat{y}) \cdot \nabla=\partial / \partial z^{\prime}$. Fourier transforms are related by $k_{y}=k_{y}^{\prime}$ and $k_{x}=k_{x}^{\prime}-s z^{\prime} k_{y}^{\prime}$. Note the similarity between this expression for $k_{x}$ and the expression for $k_{r}$ in the usual ballooning transformation $k_{r}=k_{\theta} \hat{s}\left(\theta-\theta_{0}\right)$. Thus, the above coordinate transformation can be related to the usual ballooning transformation by relating $k_{\theta}=k_{y}^{\prime}, \theta_{0}=-k_{x}^{\prime} /\left(k_{y}^{\prime} \hat{s}\right.$ ) (one must be careful with modes with $k_{y}^{\prime}=0$ and finite $k_{x}^{\prime}$ - they are physically meaningful components of the fluctuations although they have an infinite $\left.\theta_{0}\right)$, and the poloidal angle is related to the distance along the field line by $\theta=z^{\prime} /(q R)$.

The surprise is that the nonlinear terms remain easy to evaluate in the new fieldline coordinate system[4]. The $\vec{E} \times \vec{B}$ nonlinearity operating on some field $A$ is usually written using the Poisson bracket notation:

$$
\hat{z} \times \nabla \Phi \cdot \nabla A=\frac{\partial \Phi}{\partial x} \frac{\partial A}{\partial y}-\frac{\partial \Phi}{\partial y} \frac{\partial A}{\partial x} \equiv\{\Phi, A\} .
$$

Transforming to the field-line coordinates yields

$$
\{\Phi, A\}=\frac{\partial \Phi}{\partial x} \frac{\partial A}{\partial y}-\frac{\partial \Phi}{\partial y} \frac{\partial A}{\partial x}=\frac{\partial \Phi}{\partial x^{\prime}} \frac{\partial A}{\partial y^{\prime}}-\frac{\partial \Phi}{\partial y^{\prime}} \frac{\partial A}{\partial x^{\prime}}
$$

This can be easily evaluated in numerical codes using finite-differences or Fast Fourier Transform techniques. This is in contrast to the usual way in which the nonlinearity is written for the ballooning representation[15]:

$$
(\hat{b} \times \nabla \Phi \cdot \nabla A)_{k_{\theta}, \theta_{0}}(\theta)=\pi \sum_{k_{\theta}=k_{\theta}^{\prime}+k_{\theta}^{\prime \prime}} \sum_{k_{\theta} \theta_{0}=k_{\theta}^{\prime} \theta_{0}^{\prime}+k_{\theta}^{\prime \prime} \theta_{0}^{\prime \prime}} \sum_{p} e^{-i n^{\prime \prime} q 2 \pi p} k_{\theta}^{\prime} k_{\theta}^{\prime \prime} \hat{s}\left(2 \pi p+\theta_{0}^{\prime}-\theta_{0}^{\prime \prime}\right) \times
$$




$$
\left[\Phi_{k_{\theta}^{\prime}, \theta_{0}^{\prime}}(\theta+2 \pi p) \cdot A_{k_{\theta}^{\prime \prime}, \theta_{0}^{\prime \prime}}(\theta)-A_{k_{\theta}^{\prime}, \theta_{0}^{\prime}}(\theta+2 \pi p) \Phi_{k_{\theta}^{\prime \prime}, \theta_{0}^{\prime \prime}}(\theta)\right]
$$

While the usual slab nonlinearity $\left(\mathbf{k}_{\perp}{ }^{\prime} \times \mathbf{k}_{\perp}{ }^{\prime \prime}\right)$ can be efficiently evaluated with FFT's, it was not obvious that the ballooning nonlinearity, with its sum over $p$, can be. As we show below. the field-line coordinate transformation can be made equivalent to the ballooning representation (with certain boundary condition in $\theta$ ), so that Eq. (9) is the fast way to evaluate Eq. (10).

Periodic radial boundary conditions are easy to implement in these field-line coordinates even in a sheared magnetic field. Perturbations in the plasma tend to be highly elongated along magnetic field lines. Because the magnetic field points in a different direction at $x=0$ than at $x=L_{x}$, it is not appropriate to impose $\mathrm{x}$-periodicity in the unprimed coordinates $\Phi(x=0, y, z)=\Phi\left(x=L_{x}, y, z\right)$ (for example. see Fig.3 of Ref.[14]). However, one can use periodicity in $x^{\prime}, \Phi^{\prime}\left(x^{\prime}=0, y^{\prime}, z^{\prime}\right)=\Phi^{\prime}\left(x^{\prime}=L_{x}, y^{\prime}, z^{\prime}\right)$, since the field-line coordinate transformation takes care of the magnetic field direction. One should note $x^{\prime}$ is not a physically periodic variable (unlike $\theta$ or $\phi$ in a torus). Instead we are assuming that the statistical properties of the fluctuations at $x=L_{x}$ are the same as at $x=0$, and that if $L_{x}$ is much larger than the radial correlation length we can can assume they are in fact identical at every instant. This "statistical periodicity" allows one to avoid edge effects associated with $\Phi=0$ boundary conditions at the edges of a bounded box. Periodic radial boundary conditions allow the self-consistent evolution of the $\left(k_{y}=0, k_{z}=0\right)$ components of the fluctuations (which were set to zero in some previous simulations in a bounded box to avoid flattening of the background gradient) which play a dominant role in the nonlinear turbulent state since they are responsible for the sheared poloidal flows (see Sec. 5). The precise choice of boundary conditions shouldn't matter much if the box is large enough so that there is a separation of time scales between the turbulence saturation time and the profile flattening time (and if the rational surfaces are close enough together[14] so that flattening around individual surfaces doesn't dominate), though periodic boundary conditions should be a bit more efficient by allowing a somewhat smaller box.

Periodicity in the $y^{\prime}$ direction is the same as in the unprimed coordinates, $\Phi^{\prime}\left(x^{\prime}, L_{y}, z^{\prime}\right)=\Phi^{\prime}\left(x^{\prime}, 0, z^{\prime}\right)$. However, the along-the-field-line periodicity assumption should be imposed in physical coordinates, not field-line coordinates directly. The reason for this is that in the $(x, y)$ plane, the computational box is twisting into a parallelogram as it follows the field lines along $z^{\prime}$. If the eddies tend to be elongated in the $x$ direction at $z=0$ (perhaps because of "ballooning" to take advantage of the bad curvature drive at $\theta=0$ ), then they should also tend to be elongated in the physical $x$ direction (not the $x^{\prime}$ direction) at $L_{z}$ (assuming $L_{z}$ is some multiple of $2 \pi q R$ ). Using the mapping from real-space to field-line coordinates $\Phi\left(x, y, L_{z}\right)=\Phi^{\prime}\left(x^{\prime}, y^{\prime}, L_{z}\right)=\Phi^{\prime}\left(x, y-s x L_{z}, L_{z}\right)$, and expanding $\Phi^{\prime}$ in Fourier components as $\Phi^{\prime}\left(x^{\prime}, y^{\prime}, z^{\prime}\right)=\sum_{k_{x}^{\prime}} \sum_{k_{y}^{\prime}} \Phi_{k_{x}^{\prime}, k_{y}^{\prime}}^{\prime}(z) \exp \left(i k_{x}^{\prime} x^{\prime}+i k_{y}^{\prime} y^{\prime}\right)$, it can be shown that the parallel periodicity assumption translates to the field-line coordinates as $\Phi_{k_{x}^{\prime}, k_{y}^{\prime}}^{\prime}\left(L_{z}\right)=\Phi_{k_{x}^{\prime}-k_{y}^{\prime} s L_{z}, k_{y}^{\prime}}^{\prime}(0)$. If $L_{z}=2 \pi q R$, this translates into the usual ballooning representation as $\Phi_{k_{\theta}, \theta_{0}}(\theta=2 \pi)=\Phi_{k_{\theta}, \theta_{0}-2 \pi}(0)$. In general, $L_{z}$ should be chosen to be several times the parallel decorrelation length rather than just $2 \pi q R$. If one follows a very thin flux tube a distance of $2 \pi q R$ along the field, it will come back to the same poloidal angle but usually a different toroidal angle (unless one is near a low-order rational surface), and it hasn't yet "bitten it's tail" [4] to require periodicity. (Careful 
convergence studies need to be done, but in practice $L_{z}=2 \pi q R$ may be sufficient. particularly if the box is thick enough in the $x^{\prime}$ and $y^{\prime}$ directions.)

In a later paper we will present the field-line-following coordinate transformation in more detail, including more details on its relation to the usual ballooning transformation. There are also similarities between this field-line transformation[4] and some other :pproaches $[21,22]$ also proposed for efficient simulations of small-scale turbulence, though in each case one needs to think carefully about the relative advantages of various approaches. and the effects of boundary conditions on correlation lengths. J.B. Taylor et al [23] have recently discussed some potentially significant subtleties which result from including the radial variation of $\omega_{*}(r) \approx \omega_{* 0}\left(1+x / L_{*}\right)$ (one should also consider $\eta_{i}$ vs. $\left.r\right)$. When Fourier-transformed, this would lead to new terms involving $-i \partial / \partial k_{x}$ which could be included in the field-line approach, though more thought perhaps needs to be given to the radial boundary conditions in this situation.

\section{Self-generated sheared rotation and nonlinear results}

We have implemented this coordinate system in 3-D nonlinear gyrofluid simulations of toroidal ITG turbulence. We present early results here; more detailed studies will follow. Fig. 2 shows typical nonlinear results from a relatively low resolution run, showing that a saturated state is only reached with the proper adiabatic electron response, $n_{e 1} / n_{0}=$ $e(\Phi-\langle\Phi\rangle) / T_{e}$, where $\langle\Phi\rangle$ is the flux-surface-averaged potential. When $n_{e 1} / n_{10}=e \Phi / T_{e}$ is used, the turbulence does not saturate: streamer-like (radially elongated) structures form and grow indefinitely. With the (usually) proper electron response $\propto \Phi-\langle\Phi\rangle$, sheared poloidal $\mathbf{E} \times \mathbf{B}$ flows (potential perturbations with $\left(k_{y}=0, k_{z}=0\right)$, but $k_{x} \neq 0$ ) are nonlinearly generated by the turbulence. These sheared flows have a stabilizing influence on the turbulence and play an important role in regulating the saturated state. However, if $n_{e 1} / n_{0}=e \Phi / T_{e}$ is used, then electrons are allowed to flow radially in response to the $\left(k_{y}=0, k_{z}=0\right)$ component of $\Phi$, thus shorting out the radial electric field associated with the sheared rotation. (This may actually occur if the magnetic fields were completely stochastic allowing rapid radial electron transport.) To see this mathematically, consider the gyrokinetic quasineutrality condition, which in our notation [3] is $n_{e}=\bar{n}_{i}+n_{i 0}\left(\Gamma_{0}-1\right) e \Phi / T_{i}$. At long wavelengths this becomes $n_{e}=\bar{n}_{i}-k_{\perp}^{2} \rho_{s}^{2} n_{e 0} e \Phi / T_{e}$. The electron response for most Fourier components is just $n_{\epsilon}=n_{e 0} e \Phi / T_{e}$, so that the quasineutrality constraint reduces to the familiar form $\Phi \propto \bar{n}_{i} /\left(1+k_{\perp}^{2} \rho_{s}^{2}\right)$. However, the $\left(k_{y}=0, k_{z}=0\right)$ component of $n_{e 1}$ should be zero so that the quasineutrality constraint becomes $\Phi_{0,0} \propto \bar{n}_{i} / k_{x}^{2} \rho_{s}^{2}$. For $k_{x}^{2} \rho_{s}^{2} \ll 1$ this gives a large enhancement of the poloidal flow. This effect had been missed in most previous ITG simulations because of limitations in the adiabatic response, or in the treatment of the $\left(k_{y}=0, k_{z}=0\right)$ mode and boundary conditions.

Since the sheared poloidal flow has such a strong effect on the turbulence, it is important to include all of the proper drives and sinks for this flow. Poloidal flows are damped only weakly by collisions in slab geometry, and we have observed some cases where the flows grow to very large amplitudes causing complete stabilization of the turbulence for a long time, and leading to a bursting behavior in the turbulence[14]. In toroidal geometry, neoclassical poloidal flow damping may play an important role (work in progress), but we have found that the toroidal drifts of particles off of their 

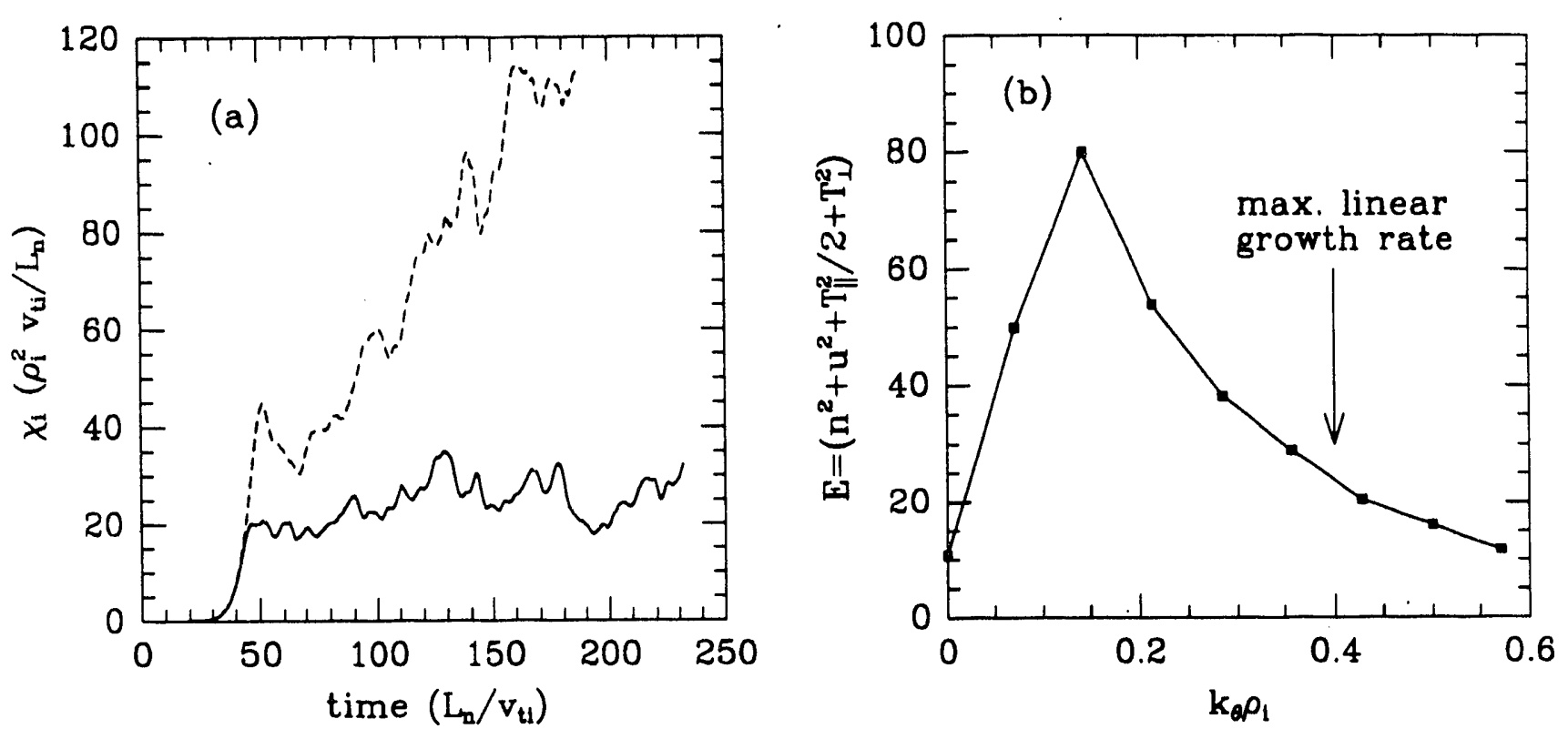

Figure 2. 3-D nonlinear gyrofluid simulations of toroidal ITG turbulence for $\eta_{i}=4, \hat{s}=1.5, L_{n} / R=0.4, T_{i}=T_{e}$, and $q=2$. (a) $\chi_{i}$ vs. timie for $n_{e} / n_{0}=e \Phi / T_{e}$ (dashed) and $n_{e} / n_{0}=e(\Phi-\langle\Phi\rangle) / T_{e}$ (solid). A saturated state is reached only when self-generated poloidal flows are allowed. (b) Time averaged saturated energy spectrum vs. $k_{\theta}$ for $n_{e} / n_{0}=e(\Phi-\langle\Phi\rangle) / T_{e}$, showing that the spectrum is downshifted from the fastest growing linear mode.

flux surfaces (modeled by the $\omega_{d}$ and $\left|\omega_{d}\right|$ terms of the gyrofluid equations of Sec. 3) cause enough smoothing of the radial density perturbations that the radial electric field does not grow too large and steady state turbulence is achieved. This is a banana-orbitaveraging effect which is analogous to the smoothing provided by gyro-orbit-averaging. Further study of the level of accuracy of our models of these toroidal effects needs to be carried out.

The generation of poloidal flow and subsequent reduction in fluctuation levels is similar to that observed in simulations by Hasegawa and Wakatani[24] for resistive drift waves and by Carreras et al.[25] for resistive pressure gradient driven turbulence, and predicted to play an important role in generating $\mathrm{L}$ to $\mathrm{H}$-mode transitions by Diamond and Kim.[26] These earlier works tended to emphasize the edge, while the present results suggest that this may also be an important effect in the plasma interior, and for all modes with near-adiabatic electron response.

The time averaged energy spectrum is shown in Fig. $2 \mathrm{~b}$ for the saturated case in Fig. 2a. Although the maximum linear growth rate is at $k_{\theta} \rho_{i} \approx 0.4$, the peak in the energy spectrum is shifted to longer wavelength. This is in agreement with general trends observed in BES measurements[20], but detailed comparisons need to be done for the actual experimental parameters vs. radius (the peak $k_{\theta} \rho_{i}$ varies with minor radius in the BES measurements). Future simulations will investigate the processes which determine the dominant scale of the fluctuations. 


\section{Conclusions}

Recent advances in gyrofluid modeling include (1) a better understanding of the convergence properties of higher order fluid moment equations and of why a limited number of fluid moments may be inadequate for some nonlinear processes, (2) an extension of the toroidal gyrofluid equations to 6 moments, including CGL-like terms from the magnetic mirroring force responsible for trapped particles, (3) the implementation of a toroidal 3-D nonlinear gyrofluid code using a field-line coordinate system for efficient representation of small scale turbulence, (4) the observation of the major role that self-generated sheared flows play in determining the saturated turbulent state. Future work is needed to (1) study further the analytic weak-turbulence limit where difficulties have been observed for lower-frequency $\left(\omega \ll k_{\|} v_{t i}\right)$ ITG modes very near marginal stability[12], (2) study the accu:acy of gyrofluid models of various neoclassical effects which may affect the self-generated sheared flow, (3) carry out higher resolution nonlinear simulations and look for what controls the long wavelength scale of the spectrum, (4) perform careful nonlinear comparisons with gyrokinetic particle simulations, and (5) compare with experimental measurements of turbulence and transport in tokamak plasmas, our ultimate goal.

\section{Acknowledgments}

It is a pleasure to acknowledge R.E. Waltz for many interesting discussions and a fruitful collaboration on the original extension to include toroidal drift resonances. We also thank G.D. Kerbel (who also provided illuminating visualizations), L. Chen, T.S. Hahm, and N. Mattor for enlightening discussions and feedback. This work is part of the Numerical Tokamak Project. Computational resources were provided at the National Energy Research Supercomputer Center, and at the Advanced Computing Lab at Los Alamos National Laboratory. This work was supported by USDoE Contract No. DEAC02- $76-\mathrm{CHO} 3073$.

\section{References}

[1] HAMMETt G W and PERKINS F W 1990 Phys. Rev. Lett. 643019

[2] HAMMETT G W, DORLAND W and PERKINS F W 1992 Phys. Fluids B 42052

[3] DORLAND W and HAMMETt G W 1993 Phys. Fluids B 5812

[4] COWLEY S C, KULSRUD R M, and SUDAN R 19S1 Phys. Fluids B 32767

[5] SMITH S A and HAMMETT G W, 1993 manuscript in preparation

[6] ARMSTRONG T P, HARDING R C, KNORR G, and MONTGOMERY D 1970 Methods in Computational Physics 929

[7] GRANT F C and FEIX M R 1967 Phys. Fluids 101356

[8] SADOWSKI W L 1967 in NASA SP-153: Symposium on Computer Simulation of Plasma and Many-Body Problems, p. 433, Clearinghouse for Federal Scientific Information, Springfield, VA, USA 
[9] STIX T H 1992 Waves in Plasmas, (New York: American Institute of Physics) 309

[10] DAVIDSON R C 1972 Nonlinear Plasma Theory (New York: Academic Press)

[11] O'NEIL T M and GOULD R W 1968 Phys. Fluids 11134

[12] MATTOR N 1992 Phys. Fluids B 43952

[13] MATTOR N and DIAMOND P H 1989 Phys. Fluids 11980

[14] DORLAND W. HAMMETT G W, HAHM T S, and BEER M A 1993 in Proc. of the U.S. -Japan Workshop on Ion Temperature Gradient Driven Turbulent Transport (New York, AIP) to be published

[15] FRIEMAN E A and CHEN L 1982 Phys. Fluids 25502

[16] LEE W W 1987 J. Comput. Phys. 72243

[17] HAHM T S 1988 Phys. Fluids 312670

[18] WALtZ R E, DOMINGUEZ R R, and HAMMETT G W 1992 Phys. Fluids B 43138

[19] DONG J Q, HORTON W and KIM J Y 1992 Phys. Fluids 41867

[20] FONCK R J, BRetZ N, COSBY G, et al 1992 Plasma Phys. Cont. Fusion 341993

[21] KOTSCHENREUTHER M and WONG H V 1991 private communication

[22] DIMITS A M 1991 in Proc. of the 14ih Int. Conf. on the Numerical Simulation of Plasmas (Annapolis, Maryland)

[23] TAYLOR J B, CONNOR J W and WILSON H R 1993 Plasma Phys. Cont. Fusion 35, This issue

[24] HASEGAWA A and WAKATANI M 1987 Phys. Rev. Lett. 591581

[25] CARRERAS B A, LYNCH V E and GARCIA L, 1991 Phys. Fluids B 31438

[26] DIAMOND P H and KIM Y B 1991 Phys. Fluids B 31626 
Dr. F. Paoloni. Univ. of Wollongong. AUSTRALIA

Prof. M.H. Bronnan, Univ. of Sydney, AUSTRALIA

Plasma Research Lob., Australian Nat Univ., AUSTRALIA

Prot. I.R. Jones, Flinders Univ, AUSTRALIA

Prot. F. Cap, Inst for Theoretical Physics, AUSTRIA

Prot. M. Heinder, Institut for Theoretiscthe Physik, AUSTRIA

Prof. M. Goossens, Astronomisch Instituut, BELGIUM

Ecole Royale Militire, Lab. do Phy. Plasmas, BELGIUM

Commission-Europeen, DG. XII-Fusion Prog., BELGIUM

Prof. R. Bouciqud, Rijksuniversibit Gent, BELGIUM

D. P.H. Sakenaka, Instiuto Freica, BRAZIL

Institu to Necione Do Posquises Especisir-INPE, BRWZIL

Documents Offico, Alomic Enorgy of Cenada Ld., CANADA

Dr. M.P. Bectynckj, MPB Tectinotogies, Inc., CANADA

Dr. H.M. Skargard, Univ. of Sackatchowan, CANADA

Prof. J. Toictmam, Univ. of Montreal, CANADA

Prof. S.R. Sreanivasen, Univ. of Calgary, CANADA

Prof. T.W. Johnston, INRS-Energie, CANADA

Dr. R. Botton, Contro canadion de husion magnétique, CANADA

Dr. C.R. James, Univ. of Abert, CANADA

Dr. P. Lukde, Komensketho Universzita, CZECHOSLOVAKIA

The Librarian, Cutham Laborabry, ENGLAND

Library, R61, Ruthertord Applaton Laboratory, ENGLAND

Mrs. S.A. Hutctinson, JET Library, ENGLAND

Dr. S.C. Sherme, Univ. of South Pacific, FIJI ISLANDS

P. Mathonen, Univ. of Helsinki, FINLAND

Prot. M.N. Buseac. Ecole Polytechnique., FRANCE

C. Nounter, Lab de Physique des Miliaux bonises. FRANCE

J. Radat CENCADARACHE - Bat 506. FRANCE

Prot. E. Economou, Unir. of Crote, GREECE

Ms. C. Rinni, Univ. of loamina, GREECE

Dr. T. Mud, Acadany Eibliographic Ser., HONG KONG

Proprint Library, Hungarian Acadomy of Sci., HUNGARY

Dr. B. DasGups, Saha inst. of Nuclaer Physics, INDIA

Dr. P. Kaw, Inst. tor Plasma Rosearch, INDLA

Dr. P. Rosenew, Iereed inst of Tectinotogy, ISPAEL

Librerien, Intemational Conter for Theo Physics, ITALY

Miss C. De Pab, Associazione EURATOMENEA, ITALY

Dr. G. Groseo, Istiuto di Ficica del Plasma, ITALY

Prof. G. Rostengni, lettuto Gas lonizzati Dot Cnr, ITALY

D. H. Yamato, Tochiba Ros a Dovel Centor, JAPAN
Prot. 1. Kawakami, Hroshma Univ., JAPAN

Prof. K. Nishikawa, Hiroshima Univ., JAPAN

Director, Japen Alomic Energy Researct Inst, JAPAN

Prof. S. Itoh, Kyustu Univ., JAPAN

Pesearct Into. CE., National instit. for Fusion Science, JAPAN

Prof. S. Tanaka, Kyoto Univ., JAPAN

Librery. Kyoto Univ., JAPAN

Prot. N. Inowe, Univ. of Tokyo, JAPAN

Secretary, Plasma Section, Electrolechnical Lab., JAPAN

S. Mori, Tectinical Advicor, LAERI, LAPAN

Dr. O. Miterai, Kumemoto inst of Tectnootogy. JAPAN

J. Hyoon-Sook, Kores Alomic Enorgy Research Inst, KOREA

D.I. Choi, The Korea Adv. Inst of Sai. \& Toch., KOREA

Prol. 8.S. Liby, Unir. of Waikato, NEW ZEALAND

In th of Physics, Chinose Acad Sa PEOPLE'S REP. OF CHINA

Librey, Inst of Plasma Physics, PEOPLE'S REP. OF CHINA

Tsinghua Univ. Library. PEOPLE'S REPUBLIC OF CHINA

Z L. S.W. Inet Physics, PEOPLE'S REPUBUC OF CHINA

Prof. J.A.C. Cebrd, Instituto Superio Tecnico. PORTUGAL

Dr. O. Potrus, AL I CUZA Univ., ROMLANIA

Dr. J. de Villiors, Fusion Studies, AEC, S. AFRICA

Prot. M.A. Helborg, Univ. of Natal, S. AFRICA

Prof. D.E. Km, Poheng Inst. of Sai. \& Tech., SO. KOREA

Prot. C.I.E.MA.T, Fusion Division Library, SPAIN

Dr. L Stonflo, Univ. Of UMEA, SWEDEN

Libry, Royd inst of Tectnology, SWEDEN

Prof. H. Wihetrneon, Chaimors Univ. of Tect., SWEDEN

Contro Phys. Dos Plasmas. Ecolo Polytich, SWITZERLAND

Bibliothook, Inst. Voor Plasma-Fysica, THE NETHERLANDS

Asst Prot. Dr. S. Cakj, Midde East Tech. Univ., TURKEY

Dr. VA. Gukhikh,Sai. Ros. Inst Eloctophys.I Apparatus, USSR

D. D.D. Ayubv, Siborian Brench of Academy of Sa., USSR

Dr. GA. Eiroov, I.V. Kurctatov Inst, USSR

Librarien, The Ukr.SSA Academy of Sciences, USSR

Dr. LM. Kovizinnykh, Inst. of General Physics, USSR

Korntorectungsandepe $\mathrm{GmbH}$, Zentralbibliothek, W. GERMANY

Bibliothok, Inst Fư Plasmatorecting. W. GERMANY

Prof. K. Sctinder, Rutr-Universitat Boctum, W. GERMANY

Dr. F. Weoner, (ASOEX), Max-Planck-Institul W. GERMANY

Librarian, Max-Planck-Institut, W. GERMANY

Prof. R.K. Janov, Inst of Ptysics, YUGOSLAVIA 

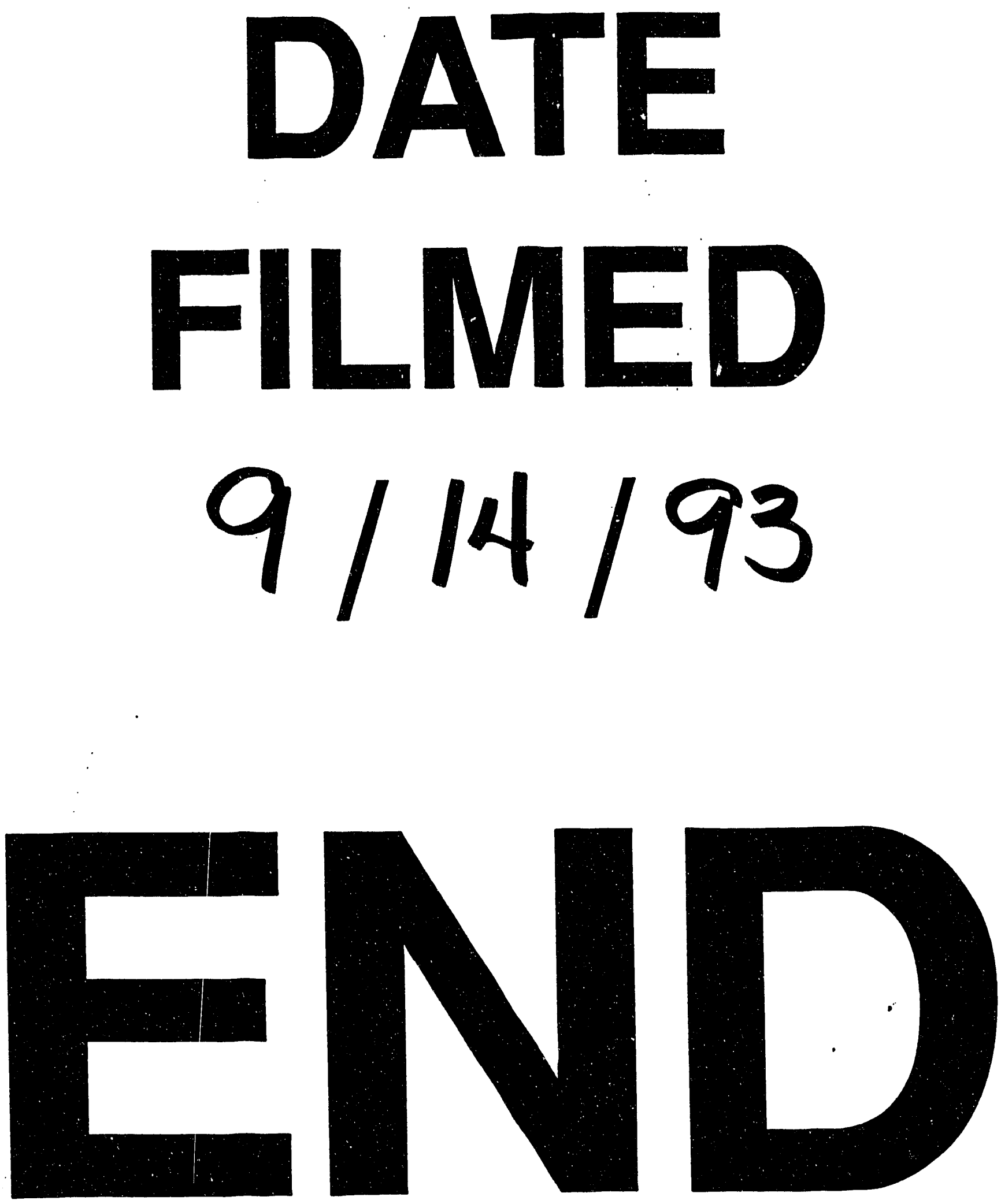
